\title{
BUFFER OCCUPANCY ANALYSIS FOR A BROADBAND POLLING-BASED WLAN
}

\author{
Ashraf S. Hasan Mahmoud, David D. Falconer, and Samy A. Mahmoud \\ ashraf@ccse.kfupm.edu.sa ${ }^{1}$, David.Falconer@sce.carleton.ca ${ }^{2}$, \\ Samy.Mahmoud@sce.carleton.ca ${ }^{2}$ \\ ${ }^{1}$ Dept of Computer Eng - King Fahd University of Petroleum and Minerals, Dhahraan, Saudi \\ Arabia \\ ${ }^{2}$ Dept of Systems and Computer Eng - Carleton University, Ottawa, Canada
}

Abstract: Driven by growing demands for high-bandwidth multimedia services, Wireless Local Area Networks (WLANs) are now being designed with access schemes catering for both synchronous and asynchronous services. A pollingbased Medium Access Control (MAC) protocol for broadband indoor WLAN is briefly presented. Analytical evaluation of the embedded queueing model is performed under some assumptions, and the wireless terminal buffer size probability mass function is derived as a function of the cell loss probability, frame length and structure, and the input traffic statistics. The analysis considers a finite buffer size terminal case, and presents statistics of buffer blocking and mean number traffic cells in buffer. The analysis considers renewal input traffic models such as the generalized batched-Bernoulli models where traffic arrivals during interservice times are statistically independent.

Key words: Wireless LANs, queueing/buffer analysis

\section{INTRODUCTION}

High demand on multimedia services and high-speed ubiquitous connectivity have driven the standardization of wireless local area networks (WLANs) standards supporting bit rates as high as $54 \mathrm{Mb} / \mathrm{s}$ [1-2]. Networks

The original version of this chapter was revised: The copyright line was incorrect. This has been corrected. The Erratum to this chapter is available at DOI: 10.1007/978-0-387-35703-4_21

D. Gaïti et al. (eds.), Network Control and Engineering for QoS, Security and Mobility II

(C) IFIP International Federation for Information Processing 2003 
such as IEEE802.11a and HIPERLAN-2 utilize advanced modulation and coding techniques to provide a shared raw bit rate of up to $54 \mathrm{Mb} / \mathrm{s}$ with provisions for both centralized and distributed medium access control (MAC) to support synchronous and asynchronous services. Studies like the on in [3] analyze the suitability of these protocols to integrate into the internet and provide the above mentioned services. More recent studies focusing on the overall performance of the access control for IEEE802.11 and HIPERLAN-2 WLANs include [4], [5], and [6]. In this paper we present a concise recounting of another WLAN design and focus on the analysis of MAC protocol for various conditions of the wireless link and the network setup.

The outline of this paper is as follows. In section 2 the proposed wireless cellular architecture is briefly recounted, with a description of the suggested MAC protocol and its parameters. In section 3, the embedded queueing model is analyzed assuming a finite buffer terminal. The characterization of assumed traffic sources is also included in the section. Results concerning the mean buffer occupancy and the buffer blocking rate are obtained in section 4 for a wide range of the operating parameters and for the traffic models considered in this paper. Finally, section 5 states the conclusions and general remarks, while the acknowledgments are presented at the end of this article.

\section{INDOOR WLAN ARCHITECTURE AND MAC PROTOCOL}

Terminals served by this network generate constant or bursty traffic over a wide range of bit rates. The network architecture should also provide service for both synchronous (e.g. voice) and asynchronous (e.g. IP data) sources. Employing a pico-cellular architecture with one port or base station per cell or domain; these ports act as access points of the WLAN and points of connectivity with the fixed infrastructure.

Aiming to support a wireless frame bit rate as high $155 \mathrm{Mb} / \mathrm{s}$, and realizing that the corresponding bandwidth is on the order of $155 \mathrm{MHz}$, direct-sequence spread-spectrum-based multiple access techniques are excluded. Furthermore, frequency-division multiple access techniques are also excluded since the original mandate requires operation in the mm-wave band. Stable radio frequency oscillators at the mm-wave band render the system cost prohibitive. The network design calls for a time-division multiple-access (TDMA)-based scheme. In addition, a low frequency reuse factor of 3 or 4 is expected given the bandwidth and hardware consideration for such broadband system design. This low reuse factor will result in a 
sever co-channel interference factor which should be reduced through the use of sectorized antennas at the base stations in a manner similar to the Altair system [7].

Traffic in each domain is organized by the domain's base station into frames of fixed duration, each consisting of both uplink and downlink traffic. The base station employs a polling scheme to serve traffic from terminals in the manner explained below. Polling-based access for wireless LANs has been analyzed in [8] and [9] in the context of IEEE 802.11. Further details of the wireless architecture of this network can be found at [10]. In the remainder of this section we focus on the structure of the TDMA frame and the subframes within, and define the parameters required for the queueing analysis to be presented in the subsequent section.

The fixed length TDMA frame of FL slots is divided into $\mathrm{S}$ sector segments or subrames. These subframes are not necessarily equal in length. During the $\mathrm{i}^{\text {th }}$ subframe, only the $\mathrm{i}^{\text {th }}$ antenna sector of the access point is used. Furthermore, the order of these subframes may be randomized from one cycle to the next so that the co-channel interference experienced by users is averaged when measured over multiple frames. The averaging of cochannel interference is essential for the proper operation of the protocol and the facilitation of the error control procedures. Each sector segment or subframe starts with a beacon envelope that announces that start of the subframe and carries status information (synchronization word, traffic condition, etc.) specific to the subframe. Wireless terminals use the $m$ registration slots that follow the beacon envelope for registration using the slotted ALOHA protocol. The number $m$ is chosen such that collisions during registration are minimal for the expected traffic load on the corresponding subframe. Employing time-division duplexing (TDD), the remaining part of the subframe is used to for downlink first and then uplink traffic to and from the users registered to that particular subframe. Figure 1 depicts the frame and subframe structure for the suggested protocol.

Synchronous traffic generating terminals may be polled at a fixed or variable rate and perhaps allocated a calculated number of transmission slots each time the polling token is sent. The polling rate should be proportional to the bit rate of the terminal and chosen to satisfy the quality of service requirement for the transported traffic. For asynchronous traffic, the polling can be optimized based on the available capacity and the statistics of the traffic. 


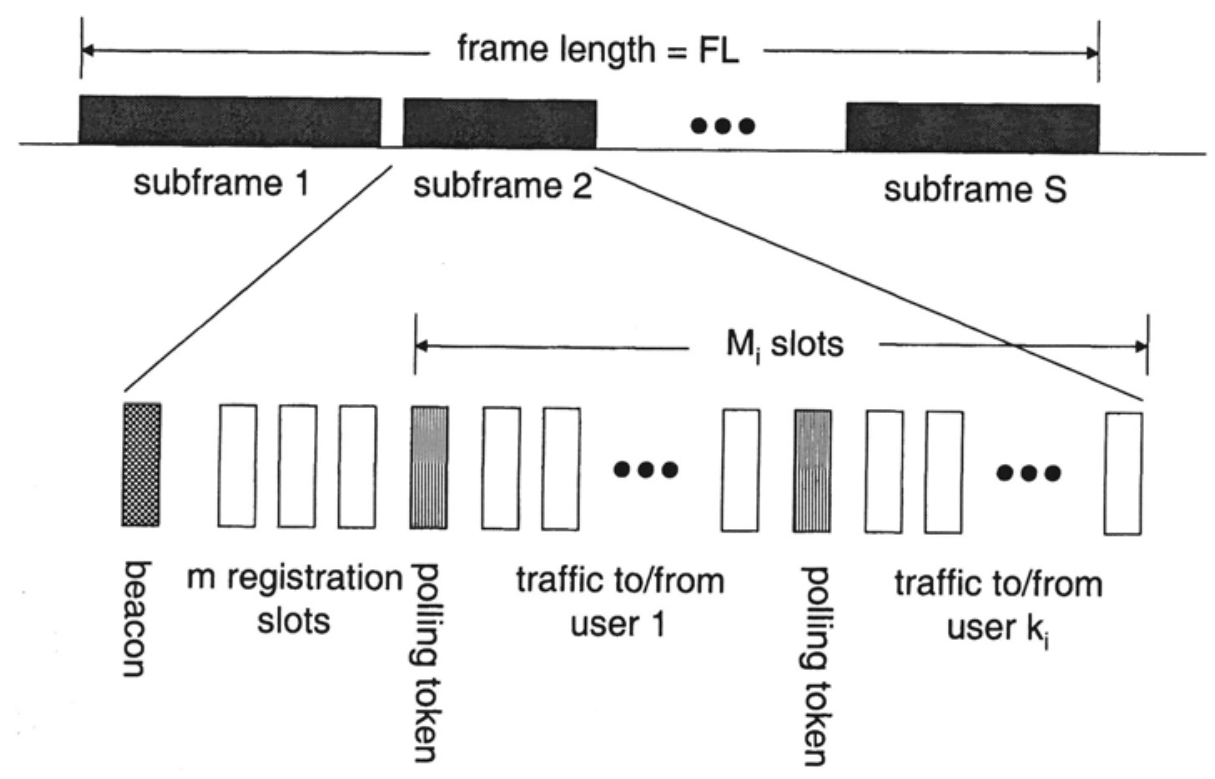

Figure 1. Frame and subframe structures for an access point.

\section{PROTOCOL QUEUEING ANALYSIS}

To carry out the buffer occupancy analysis for the proposed protocol, let us assume wireless envelopes (traffic or otherwise) are incorrectly received with probability $\alpha$ and this loss probability is independent from one time slot to the next. This is justified taking into consideration the averaging of cochannel interference and the bit/packet-level interleaving that are performed at the physical layer as a requirement for the forward-error correction procedures. A user registered with a particular subframe is sent a polling token every time its turn is reached. However, the user may or may not correctly receive the polling token. The $\mathrm{i}^{\text {th }}$ intervisit time or interservice time, $v_{\mathrm{i}}$, is defined as the duration in time slots between the $\mathrm{i}^{\text {th }}$ and the $(\mathrm{i}+1)^{\mathrm{st}}$ correctly received tokens. In [11] we have shown that for the case where traffic is spatially uniform, i.e. equally loaded subframes, the intervisit time random variable is specified by the probability generating function (PGF)

$$
V(z)=M(E(z))
$$


where $M(z)=\frac{(1-\alpha) z}{1-\alpha z} \quad$ for $\quad|\alpha z|<1 \quad$ and $\quad E(z)=\sum_{\forall i} \frac{S-|k-S|}{S^{2}} z^{i}$, $i=k(F L / S), k=1,2, . ., 2 S-1$.

Furthermore, when one user is registered per subframe, the finite queue evolution formula applies:

$$
x_{i+1}=\max \left(\begin{array}{ll}
a_{0} & i=0 \\
x_{i}-b_{i}-l_{i}+a_{i} & i=1,2, \ldots
\end{array}, K\right)
$$

where $x_{i}$ is the number of traffic payloads present in the terminal buffer upon the receipt of the $\mathrm{i}^{\text {th }}$ polling token, and $b_{i}$ is the number of traffic cell transmitted. $b_{i}$ is given by $\max \left(x_{i}, Y\right)$ where a polling gated-limited discipline is utilized. $Y$ is the maximum number of transmissions per user that can occur in any one polling cycle. $a_{i}$ is the number of traffic cells generated during the $(i+1)^{\text {st }}$ intervisit time while $l_{i}$ is the number of cells in the ARQ buffer; i.e. cells that were transmitted in the previous cycle but need to be retransmitted due to errors on the wireless channel. Finally, the variable $K$ specifies the terminal buffer size.

In [11] and in [12] it was shown that for an infinite buffer size case (i.e. $K$ is infinite), the PGF of the steady state probability distribution for the buffer occupancy random variable $x, Q(z)$, is given by

$$
Q(z)=\frac{\widehat{A}(z)\left[z^{Y} \sum_{j=0}^{Y-1} q_{j} L_{j}(z)-Q_{Y}(z) L_{Y}(z)\right]}{z^{Y}-\widehat{A}(z) L_{Y}(z)}
$$

where $\hat{A}(z)$ is the PGF specifying the traffic arrivals during the intervisit times. $L_{j}(z)=(1-\alpha+\alpha z)^{j}$ is the PGF describing the ARQ process, while $Q_{Y}(z)$ $=\Sigma q_{j} z^{j}$ for $j=0$ to $Y-1 . q_{j}$ s are the first $Y$ points of the required distribution.

Using a procedure similar to that in [11], one can show that the steady state probability distribution for the finite buffer size $K, \underline{q}$, is obtained by solving the following system

$$
(K+1) \times(K+1) Q_{(K+1) \times 1} \underline{q}={ }_{(K+1) \times 1} \underline{0}
$$

in addition to the constraint 


$$
1 \times(K+1) \underline{1}_{(K+1) \times 1} \underline{q}=1
$$

where the vectors $\underline{0}$ and $\underline{1}$ are vectors of zeros and ones respectively. The matrix $Q$, in equation (4), is give by

$$
\left[Q_{i j}\right]=\left\{\begin{array}{lc}
\sum_{k=0}^{\min (i, j)} \hat{a}_{i-k} L_{j k} & i<K, j \leq Y \\
\sum_{k=0}^{\min (K, j)} \hat{a}_{K-k} L_{j k}+\theta_{K+1} & i=K, j \leq Y \\
0 & i<K, K \leq Y<j, i<j-Y \\
\sum_{k=0}^{\min (i-(j-Y), j)} \hat{a}_{i-(j-Y)-k} L_{Y k} & i<K, K \leq Y<j, i \geq j-Y \\
\sum_{k=0}^{\min (K-(j-Y), Y)} \hat{a}_{K-(j-Y)-k} L_{Y k}+\theta_{K+1-(j-Y)} & i=K, j>Y
\end{array}\right.
$$

The variable $\hat{a}_{k}$ is equal to the probability of $k$ traffic cells arriving during the intervist time, while $\theta_{i}$ is equal to the probability of $i$ or more cells arriving during the intervist time. The ARQ term, $L_{j k}$, is the probability of having to retransmit $k$ cells out of $j$ traffic cells that were transmitted in the previous cycle. Assuming independent cell loss probability from one time slot to the next, the ARQ term is computed using

$$
L_{j k}=\left(\begin{array}{l}
j \\
k
\end{array}\right) \alpha^{k}(1-\alpha)^{j-k} \quad k \leq j
$$

The $\left[Q_{i j}\right]$ term represents the transition probability from the $j^{\text {th }}$ state into the $i^{\text {th }}$ state of the Markov chain representing this system. As illustrated in equation (6), this state transition probability is dependent on the number of cells $j$ serviced in the previous polling cycle, the fresh new arrivals as specified by $\hat{a}_{k}$, and lastly, on the ARQ term $L_{j k}$.

In the next subsection we describe the batched-Bernoulli traffic model and specify $\hat{a}_{k}$, the probability distribution of the number of cells arriving during an average intervisit time. Once this variable is characterized, given a particular WLAN frame duration and structure, and cell loss probability $\alpha$, equations (4) through (7) can be used to obtain the steady state buffer occupancy distribution. 


\subsection{Generalized Bernoulli Model Characterization}

Renewal models have been the basis for most of the results in classical queueing theory [13] because of their mathematical tractability. In discretetime queueing theory, the Bernoulli model [14] and its derivatives are typical models to consider. For the generalized Bernoulli model, a batch of one or more cells may arrive during a time slot with probability $p$ where,

$$
\beta p=\frac{R}{R_{\text {total }}}
$$

$\beta$ is the mean batch size, $R$ is the average bit rate of the traffic source, while $R_{\text {total }}$ is the total capacity of the link. Therefore, the PGF of the traffic source is given by

$$
A(z)=1-p+p G(z)
$$

where $G(z)$ is the PGF corresponding to the batch size distribution. For example for a geometrically distributed batch size with mean $\beta, G(z)$ is equal to $G(z)=\frac{(1-\zeta) z}{1-\zeta z}$ where $\zeta=1-1 / \beta$, while for a constant batch size of $\beta$ cells per batch, $G(z)=z^{\beta}$.

Finally, the required PGF for the number of cells arriving within an intervisit time, $\hat{A}(z)$, is obtained using

$$
\widehat{A}(z)=\sum_{i=0}^{\infty} \widehat{a}_{i} z^{i}=A(V(z))
$$

This PGF can be plugged into equation (6) to solve for the steady state vector $\underline{q}$.

\section{BUFFER OCCUPANCY AND BLOCKING RATE RESULTS}

Below the buffer occupancy and blocking rates are evaluated using the developed equations. Furthermore, the results are verified using simulations. The used network scenario assumes a frame rate of $155 \mathrm{Mb} / \mathrm{s}$ with 204500 bits slots per frame or 34 slots per sector segment for base stations with 6 antenna sectors. The sector segments or subframes are randomized every frame to average interference across all subframes. The gated-polling service 
scheme assumes a transmission limit $Y$ of 22 traffic cells per cycle while the data terminal generates traffic at the rate $10 \mathrm{Mb} / \mathrm{s}$. Results are shown for two values of the cell loss probability over the wireless channel: the typical value of $1 \%$ and an extreme value of $10 \%$.

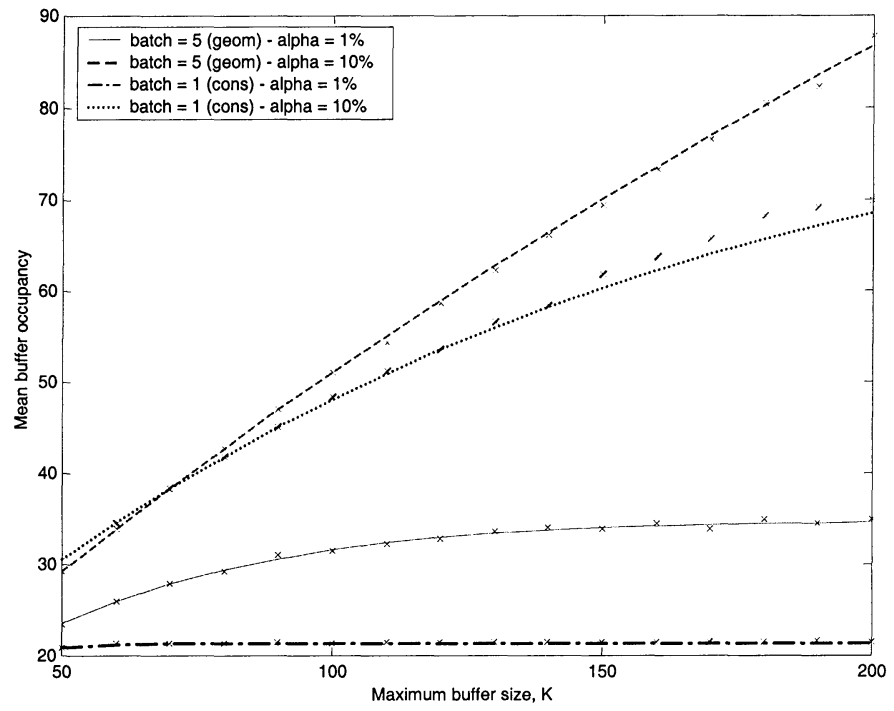

Figure 2. Mean buffer occupancy different traffic parameters - lines indicate analytical results while crosses indicate simulation results.

Figure 2 shows the mean buffer size as function of the cell loss probability, $\alpha$, and the maximum buffer size, $K$. The figure also depicts simulation results (indicated by the crosses on the graphs) which agree with the analytic ones. Simulation results were averaged over $2 \times 10^{4}$ polling cycles. The effect of ARQ term can be assessed through comparing curves for the same traffic model but for different $\alpha$ values, while the effect of the model burstiness can be deduced from curves for the same $\alpha$. Average buffer occupancy ranges from about 20 cells for the simple Bernoulli (or batchedBernoulli with constant $\beta=1$ ) model for $\alpha=1 \%$, to about 90 cells for the burstier batched-Bernoulli model with $\beta=5$ and $\alpha=10 \%$.

Figure 3 shows the buffer blocking rate which is defined as the probability of having $K$ traffic cells in the buffer. This blocking number is the $K^{\text {th }}$ (last) element of the steady state probability distribution vector $\underline{q}$ computed by equations (4) and (5). As noted in the figure the blocking rate decreases as the buffer size $K$ increases. The rate of decrease is much faster for low cells loss probability curves compared to the ones with the high $\alpha$. 
Furthermore, the figure indicates that $\alpha$ seems to play a more significant role in determining the blocking rate compared to the traffic model. The curves for $\alpha=10 \%$ for the batched-Bernoulli and the simple Bernoulli are both distinctly greater than those for $\alpha=1 \%$. As mentioned earlier, the cell loss probability determines the contribution of the ARQ term in the queue evolution formula stated in equation (2).

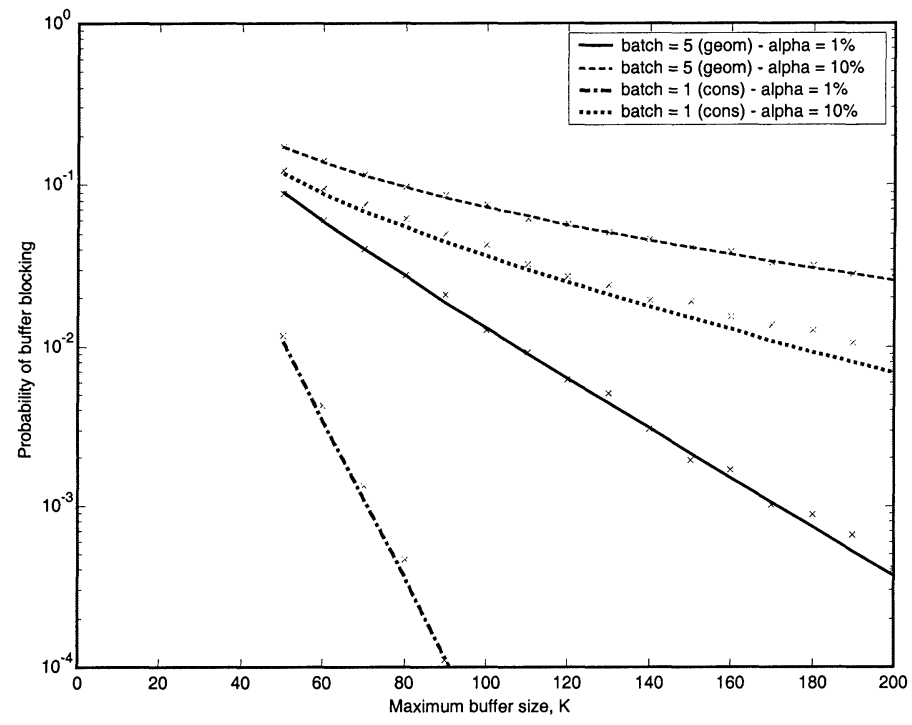

Figure 3. Buffer blocking probability for different traffic parameters - lines indicate analytical results while, crosses indicate simulation results.

\section{CONCLUSIONS AND REMARKS}

This paper presented a brief recounting of the multiple access method designed for a broadband indoor wireless local area network. The pollingbased access together with the random scheduling of subframes within the fixed length frame allow the system to operate in a relatively high interference environment. We presented and solved the embedded queueing model taking into account the wireless channel impairments, the frame structure, the service discipline, and the traffic arrival process. Furthermore, the analyzed model assumed a finite buffer size of $K$ cells at most. Analytic and simulation results for both the mean buffer size and the traffic blocking 
rate were presented depicting the effect of both the traffic model and the transmission loss probability.

\section{ACKNOWLEDGEMENTS}

The original system design was supported by a grant from the Canadian Institute for Telecommunications Research (CITR) under the NCE program of the Government of Canada from for the period from 1993 till 1996. Furthermore, the author acknowledges King Fahd University of Petroleum and Minerals, Dharaan, Saudi Arabia for support.

\section{REFERENCES}

[1] R. van Nee, et al., "New High-Rate Wireless LAN Standards," IEEE Communications Magazine, December 1999, pp. 82-88.

[2] C. Heegard, J.T. Coffey, S. Gummadi, P.A. Murthy, R. Provencio, E.J. Rossin, S. Schrum, and M.B. Shoemake, "High-Performance Ethernet," IEEE Communications Magazine, November 2001, pp. 64-73.

[3] A. Zahedi, and K. Pahlavan, "Capacity of a wireless LAN with voice and data services," IEEE Transactions on Communications, Vol. 48, No. 7, Jul 2000, pp. 1160 -1170.

[4] Haitao Wu, et al., "Performance of Reliable Transport Protocol over IEEE 802.11 Wireless LAN: Analysis and Enhancement," IEEE Infocom 2002, New York, USA, June 2002, Vol. 2, pp. 599-607.

[5] A. Grilo, Mario Nunes, "Performance Evaluation of IEEE 802.11E," PIMRC 2002, Vol. 1, pp. 511-517.

[6] Angela Doufexi, et al., "A Comparison of the HIPERLAN/2 and IEEE 802.11a Wireless LAN Standards,” IEEE Communications Magazine, May 2002, pp. 172-180.

[7] D. Buchholtz, P. Odlyzko, M. Taýlor, and R. White, "Wireless In-Building Network Architecture and Protocols," IEEE Networking Magazine, Vol. 5, No. 6, Nov. 1991, pp. 3138.

[8] M. Veeraraghavan, N. Cocker, T. Moors, "Support of voice services in IEEE 802.11 wireless LANs," IEEE Infocom 2001, Alaska, USA, April 2001, Vol. 1, pp. 488-497.

[9] Anders Lindgren, Andreas Almquist, and Olov Schelén, "Quality of Service Schemes for IEEE 802.11 Wireless LANs - An Evaluation," Mobile Networking and Applications (MONET), Vol. 8, No. 3, June 2003, pp. 223-235.

[10] A.S. Hasan Mahmoud, D.D. Falconer, and S.A. Mahmoud, "A Multiple Access Scheme for Wireless Access to Broadband ATM LAN Based on Polling and Sectorized Antennas," IEEE Journal on Selected Areas in Communications, Vol. 14, No. 4, May 1996, pp. 596-608.

[11] A.S. Hasan Mahmoud, D.D. Falconer, S.A. Mahmoud, "Analysis of a Multiple Access Scheme for Broadband Indoor Wireless ATM LAN,"Globecom'97/CTMC, Phoenix, Arizona, USA.

[12] A.S. Hasan Mahmoud, Cellular Architecture for Wireless Indoor Broadband Communication Systems in the mm-waveband, Ph.D. Thesis, Department of Systems and Computer Engineering, Carleton University, Ottawa, Ontario, Canada, January 1997.

[13] L. Kleinrock, Queueing Systems - Volume I: Theory, John Wiley \& Sons, 1975.

[14] T. Meisling, "Discrete-Time Queueing Theory," Operation Research 6, 1958, pp. 96105. 